\title{
Distributed Control Law for Load Balancing with Delay Adjustment and Primary Back-up Approach in Content Delivery Network
}

\author{
Nitish M. Shinde \\ ME Scholer \\ Department of Computer Engineering \\ G. H. Raisoni College of Engineering \\ Pune, India
}

\author{
S. R. Khiani \\ Assistant Professor \\ Department of Information Technology \\ G. H. Raisoni College of Engineering \\ Pune, India
}

\begin{abstract}
The distributed system characterized by the distributed systems where server nodes can come up short forever with probability of nonzero, the framework execution can be evaluated by method for the administration quality, characterized as the likelihood of serving all the task lined in the distributed framework before all the failure of nodes. A Content delivery network or Content Distribution Network $(\mathrm{CDN})$ is an expansive distributed systems of servers conveyed in various server farm crosswise over internet. The objective of CDN is to server with superior and accessibility. Complex component of $\mathrm{CDN}$ is request routing system i.e. demand for content to the suitable server focused around a particular set of parameters. Load-balancing issues emerge in numerous applications in any case; in particular, they assume an exceptional part in the operation of parallel and distributed registering systems. Load balancing manages parceling a project into littler assignments that can be executed simultaneously and mapping each of these assignments to computational sources such a processor or a machine. By creating methods that can outline tasks to processors in a manner that adjusts out the load, the aggregate handling time will be decreased with enhanced processor usage. The proposed framework will actualize the model focused around worldwide balancing that will similarly adjust the appeals in framework queue which additionally considers different delay modification plan and methods for backup with arbitrary crash of nodes or failure. This paper propose load balancing algorithm to improve stability, scalability, fault tolerance and delay adjustment.
\end{abstract}

\section{General Terms}

Distributed System Load Balancing.

\section{Keywords}

Content Delivery Network (CDN); control theory; request balancing.

\section{INTRODUCTION}

Content replication has developed as a better amongst the most valuable standards for the procurement of adaptable and for reliable Internet administrations [7]. With replication of content, the same information is put away at numerous geologically different servers and request by clients are then sent to one of these servers. Due to its scalability and also fault tolerance, content replication has turned into a foundation of most advanced networking architectures, including Content Delivery Networks (CDNs) and shared peer to peer $(\mathrm{P} 2 \mathrm{p})$ networks [6]. One of the key issues emerging with content replication is that of selection of server. Many content replication networks, a number of selected server nodes are in charge of mix variety of approaching customer demands and sending them to one of the servers. Given the geological span and the size of content replication networks, server determination is in a general sense not quite the same as customary load balancing issues, which more often than not expect that servers are cofound [7].

In centralized algorithms of load-balancing the worldwide load data is gathered at an only one processor, known as the central scheduler. This scheduler will make each and every load balancing choices focused around the data that is sent from different processors. In decentralized load-balancing each processor in the framework will show its load data to whatever remains of the processors with the goal that generally kept up load data tables can be upgraded. As every processor in the framework stays informed concerning the worldwide load data, load balancing choices can be made on any processor. A centralized algorithm can help a bigger framework as it forces less overhead on the framework than the decentralized algorithm. Be that as it may, centralized algorithm has lower dependability since the failure of the central scheduler will bring about the brokenness of the load balancing arrangement. So, its capacity to help small systems, a decentralized algorithm is still simpler to demonstrate. In addition, for static load-balancing issues, all data overseeing load-balancing choices is known in advance. Task will be designated at the time of arrange time as per from the earlier learning and won't be affected by the condition of the framework at the time. Then again, a dynamic load balancing technique needs to designate undertakings to the processors alertly as they arrive. A close ideal plan must be resolved on the fly such that the undertakings booked can be finished in the most limited time. As redistribution of undertakings needs to occur at the runtime, dynamic load balancing systems are more often than tough to build. On the other hand, they tend have better execution in comparison to static one.

The processing power of any distributed framework can be acknowledged by permitting its constituent Computational Elements (CEs), or nodes, to work helpfully so that substantial loads are dispensed among them in a reasonable and powerful way. Any procedure for load distribution from CEs is called load balancing (LB). A successful LB strategy guarantees ideal utilization of the distributed resources so no CE stays in an unmoving state while some other CE is being used. For the most part, the execution of LB in delay situations relies on the choice of balancing moments and in addition the level of load-exchange permitted between nodes. 
Case in point, if the network delay is negligible inside the setting of a certain application, the best execution is attained to by permitting each node to send its entire excess load to less-possessed nodes. On the other hand, in the case for which the delay in network is unreasonably substantial, it would be more judicious to diminish the measure of load exchange in order to prevent wasted time while loads are transmitting. Unmistakably, in a limited delay constrained distributedcomputing setting, the measure of load to be traded lies between these two extremes and the sum of load-exchange must be precisely chosen. A normally utilized parameter that serves to control the force of load balancing is the LB pick up.

The load-balancing procedure can be characterized by three rules: the location, the distribution and the selection rule. The location rule figures out which processors will be included in the balancing operation. Load-balancing areas can be either global or local. A worldwide area permits the balancing operation to exchange load from one processor to any of the processors in the framework, while a local area just permits balancing operations to be performed inside the set of closest neighbor processors. The distribution rule decides how to redistribute the workload among processors in the balancing area. This rule relies on upon the balancing area that is dead set by the location principle, while the choice standard settles on whether the load-balancing operation can be performed preemptively or non-preemptively. The previous may be exchanged to different processors in between the execution while, in the last, assignments must be exchanged on the off chance that they are recently made.

This paper is composed further as: Section II details about elated work studied till now. Section III presents implementation details, preliminary definitions and documentations and in addition formally expresses the IWI and MIWI mining undertakings tended to by this paper. Section IV draws conclusions and presents future work.

\section{RELATED WORK}

Throughout the most recent decades, clients have seen the development of the Internet. As an outcome, there has been a huge development in network movement, determined by quick acknowledgement of broadband access, alongside increments in framework complexity nature and content lavishness. The over-advancing nature of the Internet brings new difficulties in overseeing and conveying content to clients. As an illustration, prevalent Web benefits frequently endure congestion and bottleneck because of the vast requests made on their administrations. A sudden spike in Web content request may cause huge workload on specific Web server(s), and thus a hotspot can be produced. In the long run the Web servers are completely overpowered with the sudden increment in traffic, and the Web website holding the content gets to be incidentally unavailable. Content provider sees the Web as a vehicle to bring rich content to their clients. A diminishing in administration quality, alongside high get to postpone primarily created by long download times, leaves the clients in dissatisfaction. Organizations procure critical money related impetuses from Web-based e-business. Consequently, they are concerned to enhance the administration quality experienced by the clients while getting to their Web locales. All things considered, the past few a long time have seen a development of advances that mean to enhance content delivery and administration provisioning over the Web. At the point when utilized together, the bases supporting these advances structure another kind of network, which is regularly called to as content network [7]. In our prior work [8], [9] demonstrated that for distributed systems with reasonable irregular correspondence delays, constraining the quantity of balancing moments and improving the execution over the decision of the balancing times and the LB pick up at each one balancing moment can result in noteworthy change in processing proficiency. This inspired us to investigate the purported one-shot LB method. Specifically, once nodes are at first appointed a specific number of assignments, all nodes would together execute LB just at one recommended moment [8]. Monte Carlo studies what's more ongoing investigations directed over WLAN affirmed our idea that, for a given starting load and normal preparing rates, there exist an ideal LB pick up and an ideal balancing moment connected with the one-shot LB arrangement, which together minimize the normal general completion time. This has additionally been checked scientifically through our recovery hypothesis based numerical model [10]. In any case, this analysis has been constrained to just two nodes and has concentrated on taking care of a stating load without considering similar type of arrival loads.

Paper [4] outlines delay routing issue in the setting of dispersed networks with and without incomplete load data. Despite the fact that a general least delay routing issue is NP hard, expecting consistently distributed $\mathrm{K}$ source destination (SD) pairs at irregular, it uses a lower bound on the normal delay and exhibit by reproduction that it is tight for a certain classes of routinely deployed networks. It demonstrates that some routing in a distributed way is sufficient to accomplish asymptotically ideal load adjusting with high likelihood as K has a tendency to infinity. Keeping in mind the end goal to set such routing, in any case, every SD pair ought to know worldwide load data, which is impossible for generally networks. They proposed a novel algorithm for routing in which every SD pair picks its routing way just among a set of predefined ways. On the other hand propose an effective method for dispersed development for predefined ways that have the capacity appropriate traffic over a system. The predefined routing algorithm work in a completely circulated way with exceptionally restricted load data.

In [2], this paper displays a review of different existing load distribution models, and classifies them as far as their key functionalities for example, traffic partitioning and path selection. In view of a number of critical criteria, for example the capacity to adjust load and to keep up packet ordering alongside a few different issues, which influence system execution saw by clients and examine different cases of existing models, and after that think about and recognize their advantages and in addition deficiencies. The execution of each one model is assessed by utilizing distinctive criteria, i.e., flexibility for dynamic traffic alternately system condition changes, load adjusting and bandwidth usage efficiencies, level of flow redistribution, packet requesting preservation, communication overhead, computational complexity, and usage complexity. It is likewise clear that the execution of load circulation models generally relies on upon the features of their traffic part and way determination plans.

\section{IMPLEMENTATION DETAILS}

\subsection{System Architecture}

Following Fig. 1 shows the proposed system architecture. It shows the Back end server, Surrogate Server and client .Where after the network established all the surrogate server and Back-end server is interconnected and the Client is responsible to send Request. The main aim of proposed system is Load balancing in the network and to use scheduler for request processing and load balancing. Scheduler is 
located just after the Queue and Just before the server. And load in the queue decides whether the request is processed locally or remote server. The surrogate server is responsible for data gathering from Back End server. Load in the network is managed by forwarding the request to the least loaded server.

might again begin exchanging its methodologies to different nodes. This whole cycle may be rehashed over and over, bringing about an unsteady state. The objective of load adjusting algorithms is to defeat this issue.

\subsubsection{Scalability}

Algorithms ought to be fit for taking care of little and

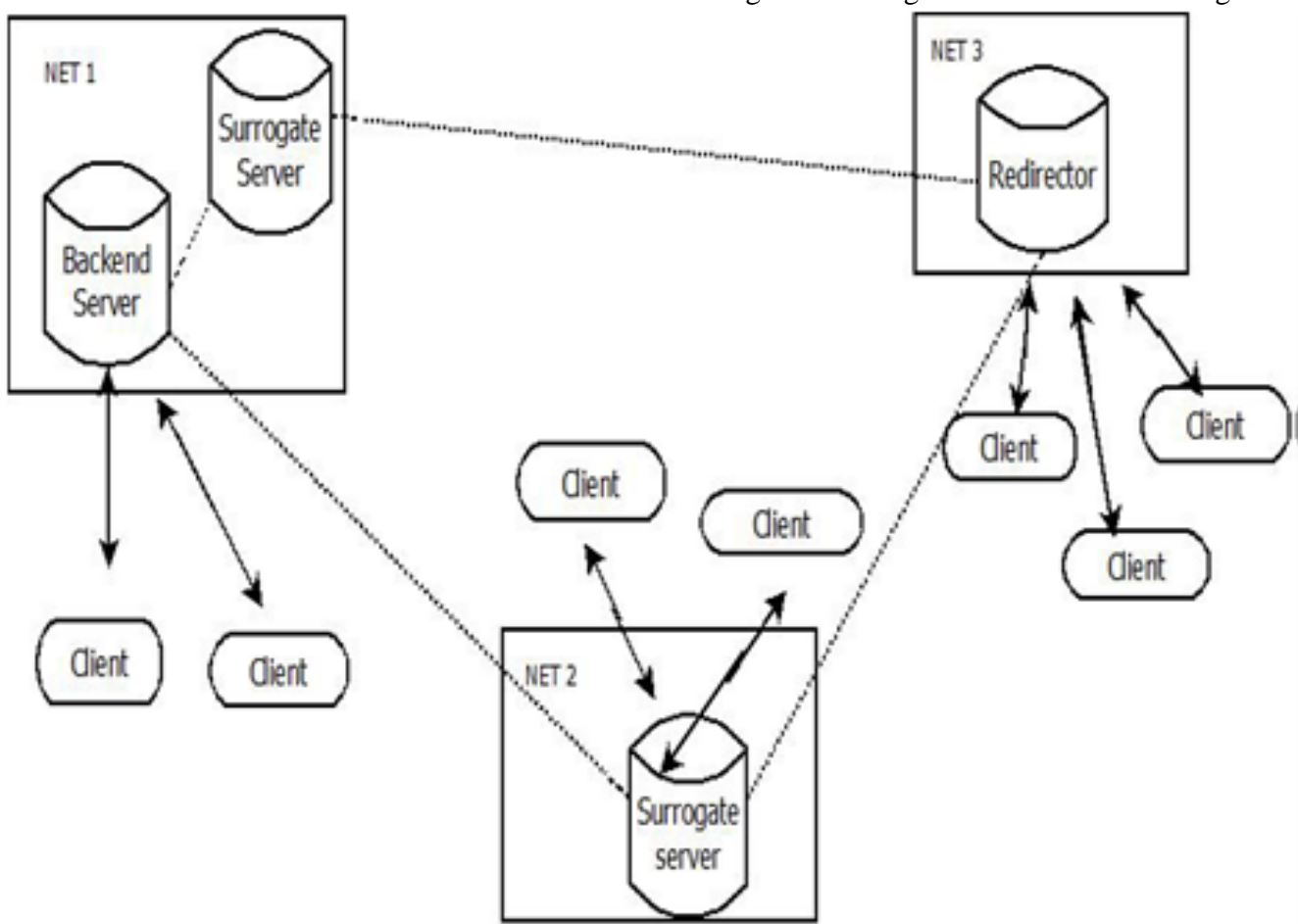

Fig 1 Proposed System Architecture

In a queue adjustment system, the scheduler is found after the queue and just before the server. The scheduler may assign request to pull out from queue to remote server relying upon the status of the framework queue. In a rate-change model, rather the scheduler is found just before the nearby line: Upon arrival of another demand, the scheduler chooses whether to assign it to the local queue or remote server. When a request is appointed to a queue, no remote rescheduling is permitted. In a hybrid adjustment procedure for load balancing, the scheduler is permitted to control both the approaching request rate at a node and the queue length. Such a methodology permits to have a more proficient load balancing in an exceptionally progressive situation; anyway in the meantime it requires a complex algorithm. The proposed systems have achieved following features by using modified approach to existing system.

\subsection{Proposed Work}

\subsubsection{Stability}

Algorithm for scheduling purpose is called unstable on the off chance that it can enter a state in which all the nodes of the framework are spreading all their times in moving methodologies without fulfilling any helpful work trying to legitimately plan the methods for better execution. This migration of unbeneficial movement is known as processor thrashing. e.g. it may like that node $\mathrm{n} 1$ and $\mathrm{n} 2$ both watched that node $\mathrm{n}$ is unmoving and afterward both offload bit of their work to node $\mathrm{n} 3$ without being offloading choice made by one another. Presently if hub $\mathrm{n} 3$ get to be overloaded because of the techniques got from both $\mathrm{n} 1$ and $\mathrm{n} 2$, then it additionally expansive system. Algorithm that settles on booking choices by first asking the workload from all the nodes and afterward selecting the most likely node as possibility for accepting the courses of action has poor scalability element. Such algorithm may work fine for little system yet gets failure when connected to huge system.

\subsubsection{Fault Tolerance}

A good algorithm ought not to be crippled by the accident of one or more nodes of the frameworks. In distributed frameworks where server nodes can come up short for all time with probability of nonzero, the framework execution can be evaluated by method for the administration dependability, characterized as the likelihood of serving all the tasks lined in the system before all the nodes failed. The system additionally allows arbitrarily detailed, load-adjusting moves to be made by the individual nodes keeping in mind the end goal to enhance the administration reliability.

\subsubsection{Delay Adjustment}

The system latency might never again be ignored the change in the load of the servers because of system delay would influence the execution of the algorithm of load balancing. At the point when the local servers have gotten the load adjusting arrangements from the other server after some system delay, the loads of the nearby servers may be altogether different and the load adjusting arrangements might never again be exact, because of the dynamic feature of DVEs.

\subsection{Algorithm}

Input: Load, Queue Buffer length. 
Output: Least loaded server

Step 1: Network Creation;

Step 2: create Queue [] at each server local and remote.

Step 3: For Each Node Find neighbor

Step 4: At every T seconds

Step 5: Update load status of neighbor's Node

Step 6: get Current Queue Length for each neighbor.

Step 7: Find neighbor with least loaded.

Step 8: End For

Step 9: request client Request

Step 10: add Request in Queue

Step 11: Start Scheduling

Step 12: send request to Local or Remote Server.

Step 13: Distribute the request to Least Loaded neighbor.

Step 14: Request Processing.

\subsection{Experimental Setup}

The system is built using Java framework (version jdk 6) on Windows platform. The Net beans (version 6.9) are used as a development tool. The system doesn't require any specific hardware to run any standard machine is capable of running the application.

\section{RESULTS AND DISCUSSION}

4.1 Datasets

In this work algorithm considers several different inputs from range 50. Table I shows training time table

\subsection{Results}

Following table I shows training time table.

Table 1. Training Time

\begin{tabular}{|l|l|l|l|l|l|l|l|}
\hline Input & $\begin{array}{l}\text { Serve } \\
\text { r1 }\end{array}$ & $\begin{array}{l}\text { Serv } \\
\text { er2 }\end{array}$ & $\begin{array}{l}\text { Serve } \\
\text { 33 }\end{array}$ & $\begin{array}{l}\text { Serve } \\
\text { r4 }\end{array}$ & $\begin{array}{l}\text { Serve } \\
\text { r5 }\end{array}$ & $\begin{array}{l}\text { Serve } \\
\text { r6 }\end{array}$ & $\begin{array}{l}\text { Serve } \\
\text { r 7 }\end{array}$ \\
\hline 50 & 20 & 15 & 33 & 45 & 22 & 60 & 75 \\
\hline 100 & 25 & 17 & 38 & 40 & 28 & 62 & 74 \\
\hline 150 & 35 & 21 & 36 & 42 & 32 & 55 & 74 \\
\hline 200 & 40 & 19 & 39 & 48 & 33 & 61 & 71 \\
\hline 250 & 26 & 23 & 41 & 51 & 28 & 63 & 78 \\
\hline 300 & 37 & 25 & 42 & 52 & 34 & 61 & 79 \\
\hline 350 & 40 & 25 & 45 & 53 & 35 & 65 & 74 \\
\hline 400 & 41 & 23 & 41 & 54 & 38 & 68 & 72 \\
\hline 450 & 39 & 19 & 50 & 49 & 39 & 62 & 71 \\
\hline 500 & 38 & 17 & 45 & 56 & 42 & 64 & 76 \\
\hline 550 & 41 & 16 & 47 & 50 & 43 & 61 & 75 \\
\hline
\end{tabular}

This graph shows the Expected result for Queue length of each server at the specific period of time. Here we are considering two factors queue length and time in seconds. This is the graph for 7 servers, the queue length is periodically updated due to request processing present in the queue. And in the proposed work the load is distributed based on least load present server. Fig. 2 shows graph for training time according to Table I.

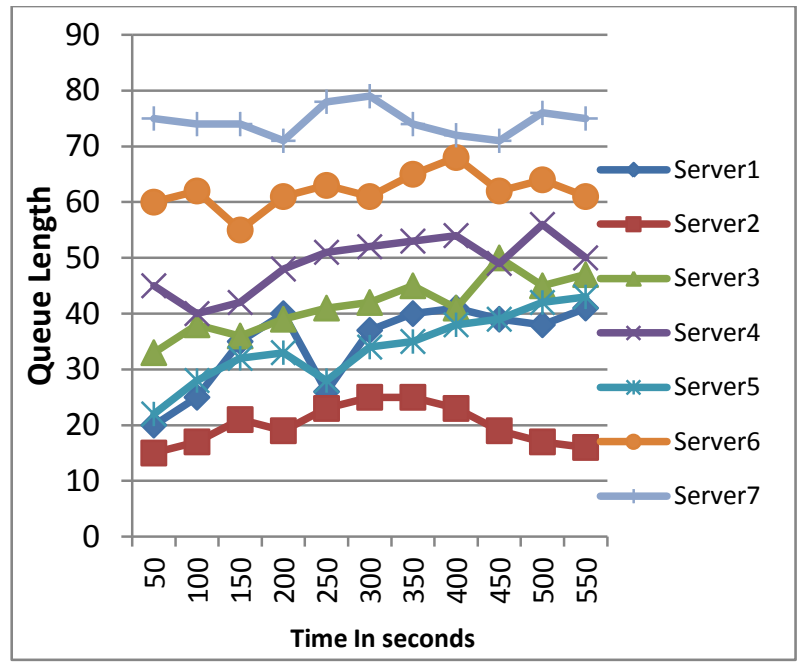

Fig2:Training Time Graph

\section{CONCLUSION}

In this proposed work, the algorithm proposes a loadbalancing technique for helpful CDN networks. We initially characterized a model of such networks focused around a flow characterization. We thus moved to the meaning of a algorithm that aims for attaining to load balancing in the system by removing nearby local queue conditions through redistribution of potential abundance traffic to the set of neighbors of the congested server. The algorithm is initially presented in now is the ideal time constant plan and afterward put in a discrete adaptation particularly imagined for its real execution and sending in an operational situation. Through the assistance of recreations, we showed the scalability, stability, fault tolerance and delay adjustment the effectiveness of our proposal, which performs the majority of the potential plan. In future work will be extended to the real execution of solution in a framework, so to arrive at a first model of a load-adjusted, CDN system to be utilized both as an evidence of-idea execution of the results got through recreations and as a play area for further research in the more non specific field of network administration.

\section{ACKNOWLEDGMENTS}

We are grateful to our project guide Mrs. Simran Khiani for her remarks, suggestions and time. Also the Head of the Department And Principal for providing all the vital facilities like for providing the required facilities, Internet access and important books, which were essential. We are also thankful to all the staff members of the Department of Information Technology of G. H. Raisoni college of Engg \& mgmt. Pune.

\section{REFERENCES}

[1] Sabato Manfredi, Member, IEEE, Francesco Oliviero, Member, IEEE, and Simon Pietro Romano, Member, IEEE,"A Distributed Control Law for load balancing in Content Delivery Network", IEEE/ACM TRANSACTIONS ON NETWORKING, VOL. 21, NO. 1, FEBRUARY 2013.

[2] Sang-Woon Jeon, "Fully Distributed Algorithms for Minimum Delay Routing Under Heavy Traffic", IEEE TRANSACTIONS ON MOBILE COMPUTI NG, VOL. 13, NO. 5, MAY 2014.

[3] Jasma Balasangameshwara and Nedunchezhian Raju "Performance- Driven Load Balancing with a PrimaryBackup Approach for Computational Grids with Low 
Communication Cost and Replication Cost" in IEEE TRANSACTIONS ON COMPUTERS, VOL. 62, NO. 5, MAY 2013

[4] Yunhua Deng and Rynson W.H. Lau "'On Delay Adjustment for Dynamic Load Balancing in Distributed Virtual Environments" in IEEE TRANSACTIONS ON VISUALIZATION AND COMPUTER GRAPHICS, VOL. 18, NO. 4, APRIL 2012.

[5] Jorge E. Pezoa, Student Member, IEEE, Sagar Dhakal, Member, IEEE, and Majeed M. Hayat, Senior Member, IEEE "Maximizing Service Reliability in Distributed Computing Systems with Random Node Failures: Theory and Implementation:" in IEEE TRANSACTIONS ON PARALLEL AND DISTRIBUTED SYSTEMS, VOL. 21, NO. 10, OCTOBER 2010.

[6] S. Manfredi, F. Oliviero, and S. P. Romano, "Distributed management for load balancing in content delivery networks," in Proc. IEEE GLOBECOM Workshop, Miami, FL, Dec. 2010, pp. 579583.
[7] H. Yin, X. Liu, G. Min, and C. Lin, "Content delivery networks: A Bridge between emerging applications and future IP networks," IEEE Netw., vol. 24, no. 4, pp. 5256, Jul.Aug. 2010

[8] Sumet Prabhavat,"On Load Distribution over Multipath Networks", IEEE COMMUNICATIONS SURVEYS and TUTORIALS, VOL. 14, NO. 3,THIRD QUARTER 2012.

[9] M.M. Hayat, S. Dhakal, C.T. Abdallah, J.D. Birdwell, and J. Chiasson, "Dynamic Time Delay Models for Load balancing. Part II: Stochastic Analysis of the Effect of Delay Uncertainty," Advances in Time Delay Systems, vol. 38, pp. 355-368, Springer- Verlag, 2004

[10] S. Dhakal, B.S. Paskaleva, M.M. Hayat, E. Schamiloglu, and C.T. Abdallah, "Dynamical Discrete-Time Load Balancing in Distributed Systems in the Presence of Time Delays," Proc. IEEE 